Pacific

Journal of

Mathematics

ANALOGUES OF THE EXPONENTIAL MAP ASSOCIATED WITH COMPLEX STRUCTURES ON NONCOMMUTATIVE TWO-TORI

AleXANDER POLISHChuK 


\title{
ANALOGUES OF THE EXPONENTIAL MAP ASSOCIATED WITH COMPLEX STRUCTURES ON NONCOMMUTATIVE TWO-TORI
}

\author{
AleXANDER POLISHCHUK
}

\begin{abstract}
We define and study analogues of exponentials for functions on noncommutative two-tori that depend on the choice of a complex structure. The major difference with the commutative case is that our exponentials can be defined only for sufficiently small functions. We show that this phenomenon is related to the existence of certain discriminant hypersurfaces in an irrational rotation algebra. As an application of our methods we give a very explicit characterization of connected components in the group of invertible elements of this algebra.
\end{abstract}

\section{Introduction}

We study some natural constructions for functions on noncommutative two-tori equipped with a complex structure. Recall that for every number $\theta \in \mathbb{R} \backslash \mathbb{Q}$ the algebra $A_{\theta}$ of smooth functions on the noncommutative torus $T_{\theta}$ (also known as the irrational rotation algebra) consists of expressions $\sum_{(m, n) \in \mathbb{Z}^{2}} a_{m, n} U_{1}^{m} U_{2}^{n}$, where the coefficients $a_{m, n} \in \mathbb{C}$ decrease rapidly at infinity and the multiplication is performed using the rule

$$
U_{1} U_{2}=\exp (2 \pi i \theta) U_{2} U_{1} .
$$

Given an element $\tau \in \mathbb{C} \backslash \mathbb{R}$ (as in [Polishchuk and Schwarz 2003] we will always assume that $\operatorname{Im} \tau<0$ ), we define a derivation

$$
\delta_{\tau}: A_{\theta} \rightarrow A_{\theta}: \sum a_{m, n} U_{1}^{m} U_{2}^{n} \mapsto 2 \pi i \cdot \sum_{m, n}(m \tau+n) a_{m, n} U_{1}^{m} U_{2}^{n}
$$

We consider $\delta_{\tau}$ as a complex structure on $T_{\theta}$ and denote the resulting complex noncommutative torus by $T_{\theta, \tau}$.

The main object of our study is the equation

$$
\delta_{\tau}(x)=x a
$$

MSC2000: 46L87.

Keywords: exponential map, noncommutative tori.

Supported in part by NSF grant. 
for $x \in A_{\theta}$, where $a \in A_{\theta}$ is given. In the commutative case this equation is clearly related with the exponential map on smooth functions. It turns out that there is a local analogue of this map for $A_{\theta}$. However, in the noncommutative case there seem to be serious reasons why the exponential map does not extend to all functions. For example, we show that $(0-1)$ has a nonzero solution if and only if $\operatorname{tr}(a) \in 2 \pi i(\mathbb{Z}+\mathbb{Z} \tau)$, where $\operatorname{tr}\left(\sum a_{m, n} U_{1}^{m} U_{2}^{n}\right)=a_{0,0}$ - see Corollary 3.7 - but these solutions are not necessarily invertible in $A_{\theta}$, as they are in the commutative case.

The study of Equation (0-1) turns out to be closely related to the study of holomorphic structures on the trivial holomorphic bundle over $T_{\theta, \tau}$. By a holomorphic bundle on $T_{\theta, \tau}$ we mean a right projective module $E$ over $A_{\theta}$ equipped with a $\delta_{\tau}$-connection, i.e., a linear map $\bar{\nabla}: E \rightarrow E$ satisfying the Leibniz rule

$$
\bar{\nabla}(e a)=\bar{\nabla}(e) a+e \delta_{\tau}(e) .
$$

The category $\mathscr{C}$ of holomorphic bundles on $T_{\theta, \tau}$ was studied in [Polishchuk and Schwarz 2003] and [Polishchuk 2004]. The main result of the latter paper identifies $\mathscr{b}$ with the heart of a certain $t$-structure on the derived category of coherent sheaves on the elliptic curve $\mathbb{C} /(\mathbb{Z}+\mathbb{Z} \tau)$. This leads to a classification of holomorphic bundles on $T_{\theta, \tau}$ up to isomorphism. We apply this classification to the study of solutions of (0-1), and in particular to the question of existence of a solution $x \in A_{\theta}^{*}$, where $A_{\theta}^{*} \subset A_{\theta}$ is the set of invertible elements. The main result here is Theorem 3.6, which states that the map

$$
A_{\theta}^{*} / \mathbb{C}^{*} \rightarrow A_{\theta}: x \mapsto x^{-1} \delta_{\tau}(x)
$$

identifies $A_{\theta}^{*} / \mathbb{C}^{*}$ with $\Omega_{\tau}+2 \pi i(\mathbb{Z}+\mathbb{Z} \tau)$, where $\Omega_{\tau}$ is a dense open subset in the hyperplane $H=\left\{a \in A_{\theta}: \operatorname{tr}(a)=0\right\}$. More precisely, we prove that $\Omega_{\tau}$ is the complement to the discriminant hypersurface $\Theta_{\tau} \subset H$ consisting of $a \in H$ such that the equation

$$
\delta_{\tau}(x)+a x-x a=0
$$

has a nontrivial solution $x \in H$. We call it a hypersurface since it coincides with the zero locus of a global holomorphic section of a holomorphic line bundle over $A_{\theta}$, induced by Quillen's determinant line bundle on the space of Fredholm operators of index zero. We study the structure of $\Theta_{\tau}$ in more detail, presenting some results that make us believe that $\Theta_{\tau}$ should be irreducible in an appropriate sense.

As a byproduct of our study we deduce the following statement about the structure of the group $A_{\theta}^{*}$. It is well known that the group of connected components of $A_{\theta}^{*}$ can be identified with $\mathbb{Z}^{2}$. We prove that for every $x \in A_{\theta}^{*}$ one has $\operatorname{tr}\left(x^{-1} \delta_{\tau}(x)\right) \in$ $2 \pi i(\mathbb{Z}+\mathbb{Z} \tau) \subset \mathbb{C}$ and that the map $x \mapsto \operatorname{tr}\left(x^{-1} \delta_{\tau}(x)\right)$ induces an isomorphism on the groups of connected components. 
The paper is organized as follows. In Section 1 we study formal exponential maps for $A_{\theta}$. First we present the construction of a map

$$
\boldsymbol{E}_{l}(\tau, \cdot): A_{\theta} \llbracket t \rrbracket / \mathbb{C} \llbracket t \rrbracket \rightarrow 1+t A_{\theta} \llbracket t \rrbracket
$$

that specializes to $a \mapsto \exp (t a) / \operatorname{tr}(\exp (t a))$ in the commutative case. In Section 2 we construct and study the normalized map

$$
\operatorname{Exp}_{l}(\tau, \cdot): A_{\theta} \llbracket t \rrbracket \rightarrow 1+t A_{\theta} \llbracket t \rrbracket
$$

that specializes to $\exp (t a)$ in the commutative case. In Section 3 we prove that our formal exponentials converge in a neighborhood of zero and then study logarithmic derivatives of invertible elements and the discriminant hypersurface.

\section{Formal exponentials}

Notation. For every $v=(m, n) \in \mathbb{Z}^{2}$ we set

$$
U_{v}=\exp (-\pi i \theta m n) U_{1}^{m} U_{2}^{n} .
$$

We have the product rule

$$
U_{v} \cdot U_{v^{\prime}}=\exp \left(2 \pi i\left\langle v, v^{\prime}\right\rangle\right) U_{v+v^{\prime}}
$$

in $A_{\theta}$, where for $v^{\prime}=\left(m^{\prime}, n^{\prime}\right)$ we set

$$
\left\langle v, v^{\prime}\right\rangle=\left\langle v, v^{\prime}\right\rangle_{\theta}=\frac{1}{2} \theta\left(m n^{\prime}-m^{\prime} n\right) .
$$

For an element $a=\sum_{v \in \mathbb{Z}^{2}} a_{v} U_{v} \in A_{\theta}$ we define the support of $a$ as the set of all $v \in \mathbb{Z}^{2}$ such that $a_{v} \neq 0$. Elements supported on a fixed rank-1 subgroup $\mathbb{Z} \subset \mathbb{Z}^{2}$ form a commutative subalgebra in $A_{\theta}$.

Recall that $A_{\theta}$ is equipped with the $\mathbb{C}$-antilinear antiinvolution $*$ defined by $\left(U_{1}\right)^{*}=U_{1}^{-1},\left(U_{2}\right)^{*}=U_{2}^{-1}$. For $v \in \mathbb{Z}^{2}$ we have $\left(U_{v}\right)^{*}=U_{-v}$; hence for $a=$ $\sum_{v \in \mathbb{Z}^{2}} a_{v} U_{v}$ we get $a^{*}=\sum_{v} a_{v}^{*} U_{v}$, where $a_{v}^{*}=\overline{a_{-v}}$.

Define the homomorphism

$$
\iota: \mathbb{Z}^{2} \rightarrow \mathbb{C}, \quad(m, n) \mapsto 2 \pi i(m \tau+n) .
$$

For every $v \in \mathbb{Z}^{2}$, we have $\delta_{\tau}\left(U_{v}\right)=\iota(v) \cdot U_{v}$.

First construction. Our formal exponentials will live in the ring $A_{\theta} \llbracket t \rrbracket$, where $t$ is a formal variable commuting with $A_{\theta}$. We extend $\delta_{\tau}$ to a $\mathbb{C} \llbracket t \rrbracket$-linear derivation of $A_{\theta} \llbracket t \rrbracket$ and the trace to a $\mathbb{C} \llbracket t \rrbracket$-linear functional tr : $A_{\theta} \llbracket t \rrbracket \rightarrow \mathbb{C} \llbracket t \rrbracket$.

Theorem 1.1. For every $\tau \in \mathbb{C} \backslash \mathbb{R}$ there exists a unique map

$$
\boldsymbol{E}_{l}(\tau, \cdot): A_{\theta} \llbracket t \rrbracket / \mathbb{C} \llbracket t \rrbracket \rightarrow 1+t A_{\theta} \llbracket t \rrbracket
$$

satisfying $\delta_{\tau}\left(\boldsymbol{E}_{l}(\tau, a)\right)=t \boldsymbol{E}_{l}(\tau, a) \cdot \delta_{\tau}(a) \quad$ and $\quad \operatorname{tr}\left(\boldsymbol{E}_{l}(\tau, a)\right)=1$. 
We need the following purely algebraic statement.

Lemma 1.2. Let $A$ be an associative algebra over $\mathbb{Q}, M$ an A-bimodule, and $d: A \rightarrow M$ a derivation. Assume that $a_{1}, \ldots, a_{n}$ are elements of $A$ such that

$$
d\left(a_{i}\right)=a_{i-1} d\left(a_{1}\right) \quad \text { for } i=2, \ldots, n .
$$

Then

$$
a_{n} d\left(a_{1}\right) \in[A, M]+d(A),
$$

where $[A, M] \subset M$ is the linear span of the elements of the form $[a, m]=$ am $-m a$ for $a \in A, m \in M$.

Proof. For every $n_{1}, \ldots, n_{k} \in[1, n]$, write

$$
\left[n_{1}, \ldots, n_{k}\right]=a_{n_{1}} \ldots a_{n_{k-1}} d\left(a_{n_{k}}\right) \quad \bmod [A, M]+d(A) .
$$

From the Leibnitz identity we get

$$
\left[n_{1}, \ldots, n_{k}\right]+c \cdot p \cdot(1, \ldots, k)=0
$$

in $A /([A, M]+d(A))$, where $c . p .(1, \ldots, k)$ denotes the terms obtained by cyclic permutation from $(1, \ldots, k)$. Since $k$ is invertible in $A$, this implies that

$$
\sum_{n_{1}+\cdots+n_{k}=n}\left[n_{1}, \ldots, n_{k}\right]=0 .
$$

On the other hand, the identity $d\left(a_{i}\right)=a_{i-1} d\left(a_{1}\right)$ implies that

$$
\left[n_{1}, \ldots, n_{k}\right]=\left[n_{1}, \ldots, n_{k-1}, n_{k}-1,1\right]
$$

for $n_{k}>1$. Set

$$
b_{k}=\sum_{n_{1}+\cdots+n_{k}=n}\left[n_{1}, \ldots, n_{k}, 1\right] .
$$

For example, $b_{1}=[n, 1]=a_{n} d\left(a_{1}\right), b_{n+1}=0$. We claim that

$$
b_{k}=-b_{k-1} \quad \text { for every } k \text {. }
$$

Indeed, using (1-2) we get

$$
b_{k}=\sum_{n_{1}+\cdots+n_{k}=n}\left[n_{1}, \ldots, n_{k-1}, n_{k}+1\right]=\sum_{n_{1}+\cdots+n_{k}=n+1, n_{k}>1}\left[n_{1}, \ldots, n_{k}\right] .
$$

Now applying (1-1) we can replace this with

$$
b_{k}=-\sum_{\substack{n_{1}+\cdots+n_{k}=n+1 \\ n_{k}=1}}\left[n_{1}, \ldots, n_{k}\right]=-b_{k-1},
$$

which proves our claim. Therefore,

$$
a_{n} d\left(a_{1}\right)=b_{1}= \pm b_{n+1}=0 \quad \text { modulo }[A, M]+d(M) .
$$


Proof of Theorem 1.1. We first show the uniqueness of $\boldsymbol{E}_{l}(\tau, a)$. If $x, y \in 1+t A_{\theta} \llbracket t \rrbracket$ satisfy $x^{-1} \delta(x)=y^{-1} \delta(y)=t \delta(a)$ then

$$
\delta\left(x y^{-1}\right)=\delta(x) y^{-1}-x y^{-1} \delta(y) y^{-1}=0,
$$

hence $x=c y$ with $c \in \mathbb{C} \llbracket t \rrbracket$. Furthermore,

$$
1=\operatorname{tr}(x)=\operatorname{tr}(c y)=c \operatorname{tr}(y)=c,
$$

so $x=y$. To show existence, write $\boldsymbol{E}_{l}(\tau, a)=1+a_{1} t+a_{2} t^{2}+\cdots$ with some $a_{i} \in A_{\theta} \llbracket t \rrbracket$ such that $\operatorname{tr}\left(a_{i}\right)=0$ (so that $\operatorname{tr}\left(\boldsymbol{E}_{l}(\tau, a)\right)=1$ as desired) and such that

$$
\delta_{\tau}\left(a_{i}\right)=a_{i-1} \delta_{\tau}(a) \text { for } i \geq 1,
$$

where $a_{0}=1$. Clearly this would imply the first equality in the conclusion of the theorem, so it is enough to show the existence of such $a_{i}$ 's. To this end we set $a_{1}=a-\operatorname{tr}(a)$ and apply Lemma 1.2 to construct $a_{i}$ for $i \geq 2$ inductively. We have

$$
\left[A_{\theta}, A_{\theta}\right] \subset H=\delta_{\tau}\left(A_{\theta}\right),
$$

where $H \subset A_{\theta}$ is the space of elements $x$ with $\operatorname{tr}(x)=0$. A similar inclusion holds for the ring of formal power series. Thus, when $a_{1}, \ldots, a_{n}$ are already constructed, Lemma 1.2 allows us to conclude that $a_{n} \delta_{\tau}(a) \in \delta_{\tau}\left(A_{\theta} \llbracket t \rrbracket\right)$, so we can define $a_{n+1}$ as the unique element satisfying $\delta_{\tau}\left(a_{n+1}\right)=a_{n} \delta_{\tau}(a)$ and $\operatorname{tr}\left(a_{n+1}\right)=0$.

Remark. In the commutative case we have

$$
\boldsymbol{E}_{l}(\tau, a)=\exp (t a) / \operatorname{tr}(\exp (t a)),
$$

thanks to the equality $\operatorname{tr}\left(\boldsymbol{E}_{l}(\tau, a)\right)=1$ of the theorem. In Section 2 we will present a way of normalizing noncommutative exponentials that reduces to the standard normalization in the commutative case.

Similarly, one can define the function $\boldsymbol{E}_{r}(\tau, \cdot)$ such that

$$
\delta_{\tau}\left(\boldsymbol{E}_{r}(\tau, a)\right)=t \delta_{\tau}(a) \cdot \boldsymbol{E}_{r}(\tau, a)
$$

and $\operatorname{tr}\left(\boldsymbol{E}_{r}(\tau, a)\right)=1$. We now extend the standard antiinvolution $*: A_{\theta} \rightarrow A_{\theta}$ to an antiinvolution of $A_{\theta} \llbracket t \rrbracket$ by $t$-linearity. From the equation $\delta_{\tau}(a)^{*}=\delta_{\bar{\tau}}\left(a^{*}\right)$ one can easily derive that

$$
\boldsymbol{E}_{r}(\tau, a)^{*}=\boldsymbol{E}_{l}\left(\bar{\tau}, a^{*}\right) .
$$

Proposition 1.3. For every $a \in A_{\theta} \llbracket t \rrbracket$ one has

$$
\boldsymbol{E}_{l}(\tau, a) \cdot \boldsymbol{E}_{r}(\tau,-a)=\boldsymbol{E}_{r}(\tau,-a) \cdot \boldsymbol{E}_{l}(\tau, a)=: s(\tau, a) \in 1+t \mathbb{C} \llbracket t \rrbracket .
$$


Proof. Indeed, set $x=\boldsymbol{E}_{l}(\tau, a), y=\boldsymbol{E}_{r}(\tau,-a)$, and $\delta=\delta_{\tau}$. Then

$$
\delta(x y)=\delta(x) y+x \delta(y)=t x \delta(a) y-t x \delta(a) y=0 .
$$

This implies that $z=x y \in 1+t \mathbb{C} \llbracket t \rrbracket$. Since $x$ is invertible in $A_{\theta} \llbracket t \rrbracket$ and $z$ commutes with $x^{-1}$, we derive that $y=z x^{-1}$ and so $y x=z=x y$.

Corollary 1.4. $\overline{\boldsymbol{s}(\tau, a)}=\boldsymbol{s}\left(\bar{\tau},-a^{*}\right)$.

Proof. We have

$$
\overline{\boldsymbol{s}(\tau, a)}=\boldsymbol{E}_{r}(\tau,-a)^{*} \boldsymbol{E}_{l}(\tau, a)^{*}=\boldsymbol{E}_{l}\left(\bar{\tau},-a^{*}\right) \boldsymbol{E}_{r}\left(\bar{\tau}, a^{*}\right)=\boldsymbol{s}\left(\bar{\tau},-a^{*}\right) .
$$

The exponential formula in terms of coefficients. We next derive an explicit formula for $\boldsymbol{E}_{l}(\tau, a)$ in terms of the coefficients of $a$.

Recall that $\iota: \mathbb{Z}^{2} \rightarrow \mathbb{C}$ is the homomorphism sending $(m, n)$ to $2 \pi i(m \tau+n)$. Define a collection of symmetric functions $f_{n}\left(v_{1}, \ldots, v_{n}\right)$ of $n$ lattice vectors recursively by setting $f_{0}=f_{1}(v) \equiv 1$ and

$$
\begin{aligned}
f_{n}\left(v_{1}, \ldots, v_{n}\right)= & \iota\left(v_{1}+\cdots+v_{n}\right)^{-1} \\
& \times \sum_{i=1}^{n} \iota\left(v_{i}\right) f_{n-1}\left(v_{1}, \ldots, \widehat{v}_{i}, \ldots, v_{n}\right) \exp \left(2 \pi i\left\langle\sum_{j \neq i} v_{j}, v_{i}\right\rangle\right)
\end{aligned}
$$

if $v_{1}+\cdots+v_{n} \neq 0$, and $f_{n}\left(v_{1}, \ldots, v_{n}\right)=0$ otherwise. It is easy to see that $f_{n}$ can also be given by

$$
f_{n}\left(v_{1}, \ldots, v_{n}\right)=\sum_{\sigma \in S\left(v_{1}, \ldots, v_{n}\right)} \frac{\iota\left(v_{\sigma(1)}\right) \ldots \iota\left(v_{\sigma(n)}\right) \exp \left(2 \pi i \sum_{i<j}\left\langle v_{\sigma(i)}, v_{\sigma(j)}\right\rangle\right)}{\iota\left(v_{\sigma(1)}\right) \iota\left(v_{\sigma(1)}+v_{\sigma(2)}\right) \ldots \iota\left(v_{\sigma(1)}+\cdots+v_{\sigma(n)}\right)},
$$

where the summation is taken over the set $S\left(v_{1}, \ldots, v_{n}\right) \subset S_{n}$ of all permutations $\sigma$ for which the denominator in the corresponding term does not vanish. When we want to stress the dependence of $f_{n}$ on $\theta$ we write $f_{n}\left(v_{1}, \ldots, v_{n} ; \theta\right)$ instead of $f_{n}\left(v_{1}, \ldots, v_{n}\right)$.

Let $\operatorname{Div}\left(\mathbb{Z}^{2}\right)_{\geq 0}$ denote the semigroup of effective divisors on $\mathbb{Z}^{2}$, i.e., of formal linear combinations $D=n_{1}\left(v_{1}\right)+\cdots+n_{k}\left(v_{k}\right)$ with $n_{i} \geq 0$ and $v_{i} \in \mathbb{Z}^{2}$. We set

$$
\begin{aligned}
\operatorname{supp} D & =\left\{v_{i}: n_{i}>0\right\}, & \operatorname{deg} D & =n_{1}+\cdots+n_{k}, \\
s(D) & =n_{1} v_{1}+\cdots+n_{k} v_{k} \in \mathbb{Z}^{2}, & D ! & =n_{1} ! \ldots n_{k} ! .
\end{aligned}
$$

Theorem 1.5. For an element $a=\sum_{v \neq 0} a_{v} U_{v} \in A_{\theta} \llbracket t \rrbracket$ such that $a_{0}=0$, one has

$$
\boldsymbol{E}_{l}(\tau, a)=\sum_{D \in \operatorname{Div}\left(\mathbb{Z}^{2}\right) \geq 0} \frac{t^{\operatorname{deg} D}}{D !} c(D) a_{D} U_{s(D)},
$$

where for $D=\left(v_{1}\right)+\cdots+\left(v_{d}\right)$, with the $v_{i}$ not necessarily distinct, we set

$$
c(D)=c_{\theta}(D):=f_{d}\left(v_{1}, \ldots, v_{d} ; \theta\right) \text { and } a_{D}=a_{v_{1}} \ldots a_{v_{d}} .
$$


Proof. Recall that $\boldsymbol{E}_{l}(\tau, a)=\sum a_{i} t^{i}$, where $\delta\left(a_{i}\right)=a_{i-1} \delta(a)$ and $\operatorname{tr}\left(a_{i}\right)=0$ for $i \geq 1$. This easily implies that

$$
a_{n}=\sum_{\substack{D \in \operatorname{Div}\left(\mathbb{Z}^{2}\right) \geq 0 \\ \operatorname{deg} D=n}} \frac{c(D)}{D !} a_{D} U_{s(D)}
$$

for some constants $c(D)$ that do not depend on $a$. The coefficients $c(D)$ are uniquely determined from this equation since the functions $a \mapsto a_{D}$ for $D \in$ $\operatorname{Div}\left(\mathbb{Z}^{2}\right)_{\geq 0}$ are linearly independent. Note also that we have $c(D)=0$ if $s(D)=0$. Comparing the coefficients with $a_{D} U_{s(D)}$ in the equation $\delta\left(a_{d}\right)=a_{d-1} \delta(a)$, where $d=\operatorname{deg} D$, we obtain

$$
\iota(s(D)) \frac{c(D)}{D !}=\sum_{v \in \operatorname{supp} D} \iota(v) \frac{c(D-v)}{(D-v) !} \exp (2 \pi i\langle s(D-v), v\rangle) .
$$

Equivalently,

$$
\iota(s(D)) c(D)=\sum_{v \in \operatorname{supp} D} n_{v} \iota(v) c(D-v) \exp (2 \pi i\langle s(D-v), v\rangle),
$$

where $n_{v}$ is the multiplicity of $v$ in $D$. This implies that $c(D)=f_{d}\left(v_{1}, \ldots, v_{d}\right)$ for $D=\left(v_{1}\right)+\cdots+\left(v_{d}\right)$.

Corollary 1.6. For every $v_{1}, \ldots, v_{n} \in \mathbb{Z}^{2}$ such that $v_{1}+\cdots+v_{n}=0$ one has

$$
\sum_{i=1}^{n} \iota\left(v_{i}\right) f_{n-1}\left(v_{1}, \ldots, \widehat{v}_{i}, \ldots, v_{n}\right)=0 .
$$

Proof. Apply equation (1-4) to $D=\left(v_{1}\right)+\cdots+\left(v_{n}\right)$.

Remark. We do not know a direct combinatorial proof of the identity of Corollary 1.6 except when $v_{1}, \ldots, v_{n}$ are sufficiently generic. See remark after Theorem 2.4.

Recall that $\left(\sum_{v} a_{v} U_{v}\right)^{*}=\sum_{v} \overline{a_{-v}} U_{v}$. Using (1-3) and Theorem 1.5, we get

$$
\boldsymbol{E}_{r}(\tau, a)=\sum_{D \in \operatorname{Div}\left(\mathbb{Z}^{2}\right) \geq 0} \frac{t^{\operatorname{deg} D}}{D !} c_{-\theta}(D) a_{D} U_{s(D)} .
$$

\section{The normalized exponential}

We now define a natural modification of $\boldsymbol{E}_{l}(\tau, \cdot)$ that reduces to the usual exponential in the commutative case.

The idea is to modify the symmetric functions $f_{n}\left(v_{1}, \ldots, v_{n}\right)$ that appear in the explicit formula for $\boldsymbol{E}_{l}(\tau, \cdot)$. Consider the rational functions

$$
R_{n}\left(x_{1}, \ldots, x_{n}\right)=\frac{x_{1} x_{2} \ldots x_{n}}{x_{1}\left(x_{1}+x_{2}\right) \ldots\left(x_{1}+\cdots+x_{n}\right)} .
$$


For a permutation $\sigma \in S_{n}$ and an $n$-tuple $x=\left(x_{1}, \ldots, x_{n}\right)$, write

$$
x^{\sigma}=\left(x_{\sigma(1)}, \ldots, x_{\sigma(n)}\right) .
$$

Recall that for an $n$-tuple of lattice vectors $\bar{v}=\left(v_{1}, \ldots, v_{n}\right)$ we have

$$
f_{n}(\bar{v})=\sum_{\sigma \in S(\bar{v})} R_{n}\left(\iota(\bar{v})^{\sigma}\right) \exp \left(2 \pi i \sum_{i<j}\left\langle v_{\sigma(i)}, v_{\sigma(j)}\right\rangle\right),
$$

where $\iota(\bar{v})=\left(\iota\left(v_{1}\right), \ldots, \iota\left(v_{n}\right)\right)$ and $S(\bar{v}) \subset S_{n}$ is the set of all permutations $\sigma$ such that $R_{n}$ is regular at $\bar{v}^{\sigma}$. We want to define a modified expression $f_{n}^{*}(\bar{v})$ that takes into account contributions from $\sigma$ such that $R_{n}$ is not defined at $\bar{v}^{\sigma}$. For this we will combine several terms corresponding to such permutations, so that the poles will cancel out.

An $n$-tuple $\bar{v}=\left(v_{1}, \ldots, v_{n}\right)$ determines a map

$$
t_{\bar{v}}: S_{n} \rightarrow \mathbb{Z}^{2} \otimes_{\mathbb{Z}} \mathbb{Z}^{2}, \quad \sigma \mapsto \sum_{i<j} v_{\sigma(i)} \otimes v_{\sigma(j)} .
$$

The fibers of $t_{\bar{v}}$ define a partition

$$
S_{n}=S(\bar{v}, 1) \sqcup \cdots \sqcup S(\bar{v}, r)
$$

of $S_{n}$. The crucial observation is the following remarkable cancellation of poles.

Proposition 2.1. The rational functions

$$
R_{S(\bar{v}, j)}(x):=\sum_{\sigma \in S(\bar{v}, j)} R_{n}\left(x^{\sigma}\right), \quad \text { for } j=1, \ldots, r,
$$

are regular at $\iota(\bar{v})$. More precisely, for every collection $1 \leq i_{1}<\cdots<i_{r} \leq n$ such that $v_{i_{1}}+\cdots+v_{i_{r}}=0$, the functions $R_{S(\bar{v}, j)}$ do not have poles along the hyperplane $x_{i_{1}}+\cdots+x_{i_{r}}=0$.

In the following discussion we view indices as elements of $\mathbb{Z} / n \mathbb{Z}$ (so $v_{n+1}=v_{1}$ ). The proof of the next lemma is straightforward:

Lemma 2.2. If $v_{1}+\cdots+v_{n}=0$ then

$$
\sum_{i<j} v_{i} \otimes v_{j}=\sum_{i<j} v_{i+1} \otimes v_{j+1},
$$

Lemma 2.3. Let $\zeta \in S_{n}$ be the cyclic permutation of order $n$. Then the function

$$
\sum_{i=0}^{n-1} R_{n}\left(x^{\zeta^{i}}\right)
$$

has no pole along $x_{1}+\cdots+x_{n}=0$. 
Proof. It suffices to prove that the function

$$
\sum_{i=1}^{n} \frac{1}{x_{i}\left(x_{i}+x_{i+1}\right) \ldots\left(x_{i}+x_{i+1}+\cdots+x_{i+n-1}\right)}
$$

has no pole along $x_{1}+\cdots+x_{n}=0$. Since

$$
x_{i}+x_{i+1}+\cdots+x_{j} \equiv-\left(x_{j+1}+x_{j+2}+\cdots+x_{i-1}\right) \quad \bmod \left(x_{1}+\cdots+x_{n}\right)
$$

we can replace the expression above with

$$
\begin{aligned}
& \left(x_{1}+\cdots+x_{n}\right)^{-1} \\
& \quad \times \sum_{i=1}^{n} \frac{(-1)^{i-1}}{\left(x_{1}+\cdots+x_{i-1}\right)\left(x_{2}+\cdots+x_{i-1}\right) x_{i-1} x_{i}\left(x_{i}+x_{i+1}\right) \ldots\left(x_{i}+\cdots+x_{n-1}\right)} .
\end{aligned}
$$

The new sum depends only on $\left(x_{1}, \ldots, x_{n-1}\right)$. Hence, our assertion is equivalent to the identity

$$
\sum_{i=1}^{n} \frac{(-1)^{i-1}}{\left(x_{1}+\cdots+x_{i-1}\right)\left(x_{2}+\cdots+x_{i-1}\right) x_{i-1} \cdot x_{i}\left(x_{i}+x_{i+1}\right) \ldots\left(x_{i}+\cdots+x_{n-1}\right)}=0 .
$$

Since the left-hand side is homogeneous of degree $-(n-1)$, it suffices to prove that it has no poles. The only possible poles are along the hyperplanes $h_{i j}=$ $x_{i}+x_{i+1}+\cdots+x_{j}=0$. Now one can check easily that for every $i \leq j$ there are exactly two terms in the sum above having poles along $h_{i j}=0$, and that their polar parts cancel out.

Proof of Proposition 2.1. We use induction in $n$ and the recursive formula

$$
R_{n}\left(x_{1}, \ldots, x_{n}\right)=R_{n-1}\left(x_{1}, \ldots, x_{n-1}\right) \frac{x_{n}}{x_{1}+\cdots+x_{n}} .
$$

Assume that the assertion holds for $n-1$. We can write $R_{S(\bar{v}, j)}$ in the form

$$
R_{S(\bar{v}, j)}=\sum_{m=1}^{n} \sum_{\sigma \in S(\bar{v}, j): \sigma(n)=m} R_{n-1}\left(x_{\sigma(1)}, \ldots, x_{\sigma(n-1)}\right) \frac{x_{m}}{x_{1}+\cdots+x_{n}}
$$

For a given $m$ let us denote $S(\bar{v}, j ; m)$ the set of $\sigma \in S(\bar{v}, j)$ such that $\sigma(n)=m$. Fix $\sigma_{0} \in S(\bar{v}, j ; m)$. Then $S(\bar{v}, j ; m) \sigma_{0}^{-1}$ leaves $m$ stable. Furthermore, it is clear that $S(\bar{v}, j ; m) \sigma_{0}^{-1}$ coincides with one piece in the partition of the set of permutations of $(1, \ldots, \widehat{m}, \ldots, n)$ associated with the $(n-1)$-tuple $\left(v_{1}, \ldots, \widehat{v}_{m}, \ldots, v_{n}\right)$. Thus, by the induction assumption all the sums

$$
\sum_{\sigma \in S(\bar{v}, j ; m)} R_{n-1}\left(x_{\sigma(1)}, \ldots, x_{\sigma(n-1)}\right)
$$


have no poles along the required hyperplanes. If $v_{1}+\cdots+v_{n} \neq 0$ this finishes the proof. It remains to consider the case when $v_{1}+\cdots+v_{n}=0$. By Lemma 2.2 in this case all the sets $S(\bar{v}, i)$ are right cosets for the subgroup $\mathbb{Z} / n \mathbb{Z} \subset S_{n}$ generated by the cyclic permutation $\zeta \in S_{n}$, where $\zeta(i)=i+1$. Therefore, by Lemma 2.3 the functions $R_{S(\bar{v}, j)}$ have no poles along the hyperplane $x_{1}+\cdots+x_{n}=0$.

We now define a rational function of $x=\left(x_{1}, \ldots, x_{n}\right)$ by

$$
R_{\bar{v}}(x)=\sum_{\sigma \in S_{n}} R\left(x^{\sigma}\right) \exp \left(2 \pi i\left\langle t_{\bar{v}}(\sigma)\right\rangle\right)=\sum_{j=1}^{r} R_{S(\bar{v}, j)}(x) \exp \left(2 \pi i\left\langle t_{\bar{v}}(S(\bar{v}, j))\right\rangle\right),
$$

where $\left\langle t_{\bar{v}}(S(\bar{v}, j))\right\rangle$ is the common value of

$$
\left\langle t_{\bar{v}}(\cdot)\right\rangle:=\langle\cdot, \cdot\rangle \circ t_{\bar{v}}: S_{n} \rightarrow \mathbb{R}
$$

on $S(\bar{v}, j)$. By Proposition 2.1 this function is regular at $\iota(\bar{v})$, so we can set

$$
f_{n}^{*}(\bar{v})=f_{n}^{*}(\bar{v} ; \theta)=R_{\bar{v}}(\iota(\bar{v})) .
$$

It is easy to check that $f_{n}^{*}$ is symmetric in $v_{1}, \ldots, v_{n}$. As before we have $f_{0}^{*}=$ $f_{1}^{*}(v) \equiv 1$.

Theorem 2.4. For an element $a=\sum_{v} a_{v} U_{v} \in A_{\theta} \llbracket t \rrbracket$ consider the series

$$
\operatorname{Exp}_{l}(\tau, a):=\sum_{D \in \operatorname{Div}\left(\mathbb{Z}^{2}\right) \geq 0} \frac{t^{\operatorname{deg} D}}{D !} c^{*}(D) a_{D} U_{s(D)} \in 1+t A_{\theta} \llbracket t \rrbracket,
$$

where for $D=\left(v_{1}\right)+\cdots+\left(v_{d}\right)$ we have set

$$
c^{*}(D)=c_{\theta}^{*}(D)=f_{n}^{*}\left(v_{1}, \ldots, v_{d} ; \theta\right) .
$$

Then

(i) $\delta_{\tau}\left(\operatorname{Exp}_{l}(\tau, a)\right)=t \operatorname{Exp}_{l}(\tau, a) \cdot \delta_{\tau}(a)$;

(ii) if $\theta=0$ or if the support of a is contained in a rank-1 subgroup $\mathbb{Z} \subset \mathbb{Z}^{2}$ then $\operatorname{Exp}_{l}(\tau, a)=\exp (t a) ;$ and

(iii) for $a \in A_{\theta} \llbracket t \rrbracket$ and $z \in \mathbb{C} \llbracket t \rrbracket$ we have

$$
\operatorname{Exp}_{l}(z+a)=\exp (t z) \cdot \operatorname{Exp}_{l}(a) .
$$

Proof. As in Theorem 1.5 the proof of first equality reduces to the identity

$$
\begin{aligned}
\iota\left(v_{1}+\cdots+v_{n}\right) & f_{n}^{*}\left(v_{1}, \ldots, v_{n}\right) \\
& =\sum_{m=1}^{n} \iota\left(v_{m}\right) f_{n-1}^{*}\left(v_{1}, \ldots, \widehat{v}_{m}, \ldots, v_{n}\right) \exp \left(2 \pi i\left\langle\sum_{j \neq m} v_{j}, v_{m}\right\rangle\right) .
\end{aligned}
$$


We claim that in fact, there is an identity between the corresponding rational functions:

$$
\begin{aligned}
& \left(x_{1}+\cdots+x_{n}\right) R_{\bar{v}}\left(x_{1}, \ldots, x_{n}\right) \\
& \quad=\sum_{m=1}^{n} x_{m} R_{\bar{v}^{(m)}}\left(x_{1}, \ldots, \widehat{x}_{m}, \ldots, x_{n}\right) \exp \left(2 \pi i\left\langle\sum_{j \neq m} v_{j}, v_{m}\right\rangle\right),
\end{aligned}
$$

where $\bar{v}^{(m)}=\left(v_{1}, \ldots, \widehat{v}_{m}, \ldots, v_{n}\right)$. Indeed, we can rewrite the left-hand side as

$$
\begin{aligned}
&\left(x_{1}+\cdots+x_{n}\right) \sum_{\sigma \in S_{n}} R_{n}\left(x^{\sigma}\right) \exp \left(2 \pi i\left\langle t_{\bar{v}}(\sigma)\right\rangle\right) \\
&=\sum_{\sigma \in S_{n}} x_{\sigma(n)} R_{n-1}\left(x_{\sigma(1)}, \ldots, x_{\sigma(n-1)}\right) \\
& \quad \times \exp \left(2 \pi i\left(\sum_{i<j<n}\left\langle v_{\sigma(i)}, v_{\sigma(j)}\right\rangle+\left\langle\sum_{j \neq \sigma(n)} v_{j}, v_{\sigma(n)}\right\rangle\right)\right) \\
&=\sum_{m=1}^{n} x_{m} R_{\bar{v}^{(m)}}\left(x_{1}, \ldots, \widehat{x}_{m}, \ldots, x_{n}\right) \exp \left(2 \pi i\left\langle\sum_{j \neq m} v_{j}, v_{m}\right\rangle\right) .
\end{aligned}
$$

If $\theta=0$ or the vectors $v_{1}, \ldots, v_{n}$ belong to a rank-1 subgroup $\mathbb{Z} \subset \mathbb{Z}^{2}$, we have

$$
R_{\bar{v}}(x)=\sum_{\sigma \in S_{n}} R_{n}\left(x^{\sigma}\right) .
$$

Applying Proposition 2.1 we deduce that this function has no poles. Since it is homogeneous of degree 0 it must be a constant. To compute this constant we substitute $x_{1}=\ldots=x_{n}=1$ and obtain that in this case $R_{\bar{v}}(x) \equiv 1$. This immediately implies (ii).

To prove part (iii) it suffices to check (2-1) as a formal identity in $A_{\theta} \llbracket z, t \rrbracket$. We claim that both sides solve the same differential equation $\frac{\partial f}{\partial z}(z, t)=t f(z, t)$ with the same initial condition $f(0, t)=\operatorname{Exp}_{l}(\tau, a)$. This is clear for the right-hand side, so we just have to check this for the left-hand side. It is easy to check that

$$
\frac{1}{D !} \cdot \frac{\partial\left[(a+z)_{D}\right]}{\partial z}=\frac{(a+z)_{D-(0)}}{(D-(0)) !}
$$

if $D-(0) \geq 0$, otherwise the derivative is zero. This implies that the derivative of $\operatorname{Exp}_{l}(z+a)$ with respect to $z$ is equal to $t \operatorname{Exp}_{l}(z+a)$, as claimed.

Remark. For $n$ lattice vectors $\left(v_{1}, \ldots, v_{n}\right)$ such that $v_{1}+\cdots+v_{n}=0$ we obtain from the proof above that

$$
\sum_{m=1}^{n} \iota\left(v_{m}\right) f_{n-1}^{*}\left(v_{1}, \ldots, \widehat{v}_{m}, \ldots, v_{n}\right)=0 .
$$

If $v_{1}, \ldots, v_{n}$ are sufficiently generic this reduces to the identity of Corollary 1.6.

Corollary 2.5. If $\operatorname{Exp}_{l}(\tau, a)=\operatorname{Exp}_{l}(\tau, b)$ for some $a, b \in A_{\theta} \llbracket t \rrbracket$ then $a=b$.

Proof. Using part (i) of Theorem 2.4 we immediately deduce that $b=a+z$ for some $z \in \mathbb{C} \llbracket t \rrbracket$. Now part (iii) implies that $\exp (t z)=1$ and hence $z=0$. 
We can also define the right exponential map $\operatorname{Exp}_{r}(\tau, \cdot)$ by setting

$$
\operatorname{Exp}_{r}(\tau, a)=\operatorname{Exp}_{l}\left(\bar{\tau}, a^{*}\right)^{*}
$$

Then

$$
\delta_{\tau}\left(\operatorname{Exp}_{r}(\tau, a)\right)=t \delta_{\tau}(a) \cdot \operatorname{Exp}_{r}(\tau, a) .
$$

Using the definition of $\operatorname{Exp}_{l}(\tau, \cdot)$ we get

$$
\operatorname{Exp}_{r}(\tau, a)=\sum_{D \in \operatorname{Div}\left(\mathbb{Z}^{2}\right) \geq 0} \frac{t^{\operatorname{deg} D}}{D !} c_{-\theta}^{*}(D) a_{D} U_{s(D)} .
$$

Proposition 2.6. For every $a \in A_{\theta} \llbracket t \rrbracket$ one has

$$
\operatorname{Exp}_{l}(\tau, a)^{-1}=\operatorname{Exp}_{r}(\tau,-a) .
$$

Proof. It suffices to prove that for every divisor $D>0$ on $\mathbb{Z}^{2}$ one has

$$
\sum_{\substack{D_{1}, D_{2} \in \operatorname{Div}\left(\mathbb{Z}^{2}\right) \\ D_{1}+D_{2}=D}} \frac{(-1)^{\operatorname{deg} D_{2}}}{D_{1} ! D_{2} !} c_{\theta}^{*}\left(D_{1}\right) c_{-\theta}^{*}\left(D_{2}\right) \exp \left(2 \pi i\left\langle s\left(D_{1}\right), s\left(D_{2}\right)\right\rangle_{\theta}\right)=0 .
$$

This is equivalent to the following identity, which should hold for every $n$-tuple $\left(v_{1}, \ldots, v_{n}\right)$ of lattice vectors (where $n>0$ ):

$\begin{aligned} \sum_{I=\left\{i_{1}, \ldots, i_{r}\right\} \subset[1, n]}(-1)^{n-r} f_{r}^{*}\left(v_{i_{1}}, \ldots,\right. & \left.v_{i_{r}} ; \theta\right) f_{n-r}^{*}\left(v_{j_{1}}, \ldots, v_{j_{n-r}} ;-\theta\right) \\ & \times \exp \left(2 \pi i\left\langle v_{i_{1}}+\cdots+v_{i_{r}}, v_{j_{1}}+\cdots+v_{j_{n-r}}\right\rangle_{\theta}\right)=0,\end{aligned}$

where the sum is taken over all subsets $I=\left\{i_{1}, \ldots, i_{r}\right\}$ of $[1, n]=\{1,2, \ldots, n\}$ and we denote by $\left\{j_{1}, \ldots, j_{n-r}\right\}$ the complement to $I$. Recalling the definition of the functions $f_{n}^{*}$, we see that it suffices to prove the following identity between rational functions of $x_{1}, \ldots, x_{n}$ :

$$
\begin{aligned}
& \sum_{r=0}^{n} \sum_{\sigma \in S_{n}}(-1)^{n-r} R_{r}\left(x_{\sigma(1)}, \ldots, x_{\sigma(r)}\right) R_{n-r}\left(x_{\sigma(n)}, \ldots, x_{\sigma(r+1)}\right) \\
& \times \exp \left(2 \pi i \left(\sum_{i<j \leq r}\left\langle v_{\sigma(i)}, v_{\sigma(j)}\right\rangle_{\theta}+\sum_{i>j>r}\left\langle v_{\sigma(i)}, v_{\sigma(j)}\right\rangle-\theta\right.\right. \\
& \left.\left.+\left\langle\sum_{i \leq r} v_{\sigma(i)}, \sum_{j>r} v_{\sigma(j)}\right\rangle_{\theta}\right)\right)=0 .
\end{aligned}
$$

Due to the skew-symmetry of $\langle\cdot, \cdot\rangle_{\theta}$ we have

$$
\left\langle v, v^{\prime}\right\rangle_{-\theta}=\left\langle v^{\prime}, v\right\rangle_{\theta} .
$$


Using this observation we can rewrite our identity as

$$
\begin{aligned}
\sum_{r=0}^{n} \sum_{\sigma \in S_{n}}(-1)^{n-r} R_{r}\left(x_{\sigma(1)}, \ldots, x_{\sigma(r)}\right) R_{n-r}( & \left.x_{\sigma(n)}, \ldots, x_{\sigma(r+1)}\right) \\
& \times \exp \left(2 \pi i \sum_{i<j}\left\langle v_{\sigma(i)}, v_{\sigma(j)}\right\rangle_{\theta}\right)=0 .
\end{aligned}
$$

Thus, it is enough to prove that

$$
\sum_{r=0}^{n}(-1)^{n-r} R_{r}\left(x_{1}, \ldots, x_{r}\right) R_{n-r}\left(x_{n}, \ldots, x_{r+1}\right)=0 .
$$

This is equivalent to the identity

$$
\begin{aligned}
\sum_{i=0}^{n-1} \frac{(-1)^{i}}{x_{1}\left(x_{1}+x_{2}\right) \ldots\left(x_{1}+\cdots+x_{n-i}\right) x_{n}\left(x_{n}+\right.} & \\
& =\frac{\left.(-1)_{n-1}\right) \ldots\left(x_{n}+\cdots+x_{n-i+1}\right)}{x_{n}\left(x_{n}+x_{n-1}\right) \ldots\left(x_{n}+\cdots+x_{1}\right)} .
\end{aligned}
$$

We claim that more generally for $0<j<n$ one has

$$
\begin{array}{r}
\sum_{i=0}^{j} \frac{(-1)^{i}}{x_{1}\left(x_{1}+x_{2}\right) \ldots\left(x_{1}+\cdots+x_{n-i}\right) x_{n}\left(x_{n}+x_{n-1}\right) \ldots\left(x_{n}+\cdots+x_{n-i+1}\right)} \\
=\frac{(-1)^{j}}{x_{1}\left(x_{1}+x_{2}\right) \ldots\left(x_{1}+\cdots+x_{n-j-1}\right)\left(x_{1}+\cdots+x_{n}\right)} \\
\quad \times x_{n}\left(x_{n}+x_{n-1}\right) \ldots\left(x_{n}+\cdots+x_{n-j+1}\right)
\end{array} .
$$

This can be easily checked by induction in $j$.

Corollary 2.7. $\operatorname{tr}\left(\operatorname{Exp}_{l}(\tau, a)\right) \cdot \operatorname{tr}\left(\operatorname{Exp}_{r}(\tau,-a)\right)=\boldsymbol{s}(\tau, a)^{-1}$.

Proof. This follows from the relation $\operatorname{Exp}_{l}(\tau, a)=\operatorname{tr}\left(\operatorname{Exp}_{l}(\tau, a)\right) \boldsymbol{E}_{l}(\tau, a)$ and the similar relation for right exponentials.

Finally, we state the analogue of the equation $\exp (x) \exp (y)=\exp (x+y)$ for our exponentials.

Proposition 2.8. For every $a, b \in A_{\theta} \llbracket t \rrbracket$ there exists a unique $\varphi(a, b) \in A_{\theta} \llbracket t \rrbracket$ such that

$$
\operatorname{Exp}_{l}(a) \operatorname{Exp}_{l}(b)=\operatorname{Exp}_{l}(\varphi(a, b)+b)
$$

and

$$
\delta_{\tau}(\varphi(a, b))=\operatorname{Exp}_{l}(\tau, b)^{-1} \delta_{\tau}(a) \operatorname{Exp}_{l}(\tau, b)
$$


Proof. Since the element $\operatorname{Exp}_{l}(\tau, b)^{-1} \delta_{\tau}(a) \operatorname{Exp}_{l}(\tau, b) \in A_{\theta} \llbracket t \rrbracket$ has zero trace we can find some element $\varphi^{\prime} \in A_{\theta} \llbracket t \rrbracket$ such that

$$
\delta_{\tau}\left(\varphi^{\prime}\right)=\operatorname{Exp}_{l}(\tau, b)^{-1} \delta_{\tau}(a) \operatorname{Exp}_{l}(\tau, b) .
$$

Now one can easily check that the left-hand side of (2-2) is a solution of the equation

$$
\delta_{\tau}(x)=t x \delta_{\tau}\left(\varphi^{\prime}+b\right) .
$$

It follows that

$$
\operatorname{Exp}_{l}(a) \operatorname{Exp}_{l}(b)=f(t) \operatorname{Exp}_{l}\left(\varphi^{\prime}+b\right)
$$

for some $f(t) \in 1+t \mathbb{C} \llbracket t \rrbracket$. Writing $f(t)$ in the form $\exp (t z)$ for $z \in \mathbb{C} \llbracket t \rrbracket$ and using Theorem 2.4(iii) we conclude that (2-2) holds for $\varphi(a, b)=z+\varphi^{\prime}$. Uniqueness follows from Corollary 2.5.

Compatibility with the $\mathrm{SL}_{2}(\mathbb{Z})$-action. The group $\mathrm{SL}_{2}(\mathbb{Z})$ acts on the algebra $A_{\theta}$ by automorphisms. Namely, to an element $g=\left(\begin{array}{ll}a & b \\ c & d\end{array}\right) \in \mathrm{SL}_{2}(\mathbb{Z})$ one associates an automorphism

$$
\alpha_{g}: A_{\theta} \rightarrow A_{\theta}: U_{v} \mapsto U_{g v} .
$$

These automorphisms are compatible with derivations $\delta_{\tau}$ and the modular action of $\mathrm{SL}_{2}(\mathbb{Z})$ on $\tau$ :

$$
\alpha_{g} \delta_{\tau} \alpha_{g}^{-1}=\delta_{g \tau}
$$

where $g \tau=(a \tau+b) /(c \tau+d)$.

The only way in which the standard basis of $\mathbb{Z}^{2}$ enters into our formulae for $\boldsymbol{E}_{l}(\tau, a)$ and $\operatorname{Exp}_{l}(\tau, a)$ is through the definition of the homomorphism $\iota=\iota_{\tau}$ : $\mathbb{Z}^{2} \rightarrow \mathbb{C}$. For an element $g \in \mathrm{SL}_{2}(\mathbb{Z})$ we have

$$
\iota_{g \tau}(v)=\iota_{\tau}\left({ }^{t} g v\right) \cdot j_{\tau}(g)^{-1},
$$

where ${ }^{t} g$ is the transpose of $g$ and $j_{\tau}(g)=c \tau+d$. The functions $f_{n}^{*}\left(v_{1}, \ldots, v_{n}\right)$ and $f_{n}\left(v_{1}, \ldots, v_{n}\right)$ are homogeneous of degree 0 , so we get

$$
f_{n}^{*}\left(v_{1}, \ldots, v_{n} ; g \tau\right)=f_{n}^{*}\left({ }^{t} g v_{1}, \ldots,{ }^{t} g v_{n} ; \tau\right)
$$

and similarly for the $f_{n}$. This easily implies the following result.

Proposition 2.9. For every $a \in A_{\theta} \llbracket t \rrbracket$ and every $g \in \mathrm{SL}_{2}(\mathbb{Z})$ one has

$$
\begin{gathered}
\boldsymbol{E}_{l}\left(g^{\prime} \tau, \alpha_{g}(a)\right)=\alpha_{g} \boldsymbol{E}_{l}(\tau, a), \quad \operatorname{Exp}_{l}\left(g^{\prime} \tau, \alpha_{g}(a)\right)=\alpha_{g} \operatorname{Exp}_{l}(\tau, a), \\
\boldsymbol{E}_{r}\left(g^{\prime} \tau, \alpha_{g}(a)\right)=\alpha_{g} \boldsymbol{E}_{r}(\tau, a), \quad \operatorname{Exp}_{r}\left(g^{\prime} \tau, \alpha_{g}(a)\right)=\alpha_{g} \operatorname{Exp}_{r}(\tau, a), \\
\boldsymbol{s}\left(g^{\prime} \tau, \alpha_{g}(a)\right)=\boldsymbol{s}(\tau, a),
\end{gathered}
$$

where $g^{\prime}={ }^{t} g^{-1}$. 


\section{Convergent exponentials, logarithmic derivatives and the discriminant hypersurface}

Norms. In this section we establish notation for some of the norms we use on $A_{\theta}$.

First we have the operator algebra norm $\|\cdot\|$ on $A_{\theta}:\|a\|$ is defined to be the norm of the operator of multiplication by $a$ on the $L^{2}$-completion of $A_{\theta}$. It does not matter whether one considers left or right multiplication. Recall that the $C^{*}$ algebra $\bar{A}_{\theta}$ is defined as the completion of $A_{\theta}$ with respect to this norm.

We will also consider the $L^{2}$-norm

$$
\|a\|_{0}=\left(\sum_{v \in \mathbb{Z}^{2}}\left|a_{v}\right|^{2}\right)^{1 / 2},
$$

where $a=\sum_{v \in \mathbb{Z}^{2}} a_{v} U_{v}$. From the definition, for every $a, b \in A_{\theta}$ we have

$$
\|a b\|_{0} \leq\|a\| \cdot\|b\|_{0} .
$$

For every $s \geq 0$ we can also consider the norm

$$
\|a\|_{s}^{2}=\sum_{i=0}^{s}\left\|\delta_{\tau}^{i} a\right\|_{0}^{2} .
$$

The completion of $A_{\theta}$ with respect to this norm is the Sobolev space $W_{s}$. We have a sequence of embeddings $W_{0} \supset W_{1} \supset W_{2} \supset \cdots$. These spaces enjoy all the usual properties of the Sobolev spaces (see [Polishchuk 2004]). In particular, this sequence of spaces can be extended to one indexed over $\mathbb{Z}$, so that $W_{s}$ is dual to $W_{-s}$. Also, the intersection of all the $W_{s}$ is $A_{\theta}$ and the topology on $A_{\theta}$ is the one determined by the collection of seminorms coming from the $W_{s}$.

Finally, at one point we will use the norm

$$
\|a\|_{l^{1}}:=\sum_{v \in \mathbb{Z}^{2}}\left|a_{v}\right|
$$

For $a, b \in A_{\theta}$ we have

$$
\|a b\|_{l^{1}} \leq \sum_{v \in \mathbb{Z}^{2}}\left\|a_{v} U_{v} b\right\|_{l^{1}}=\sum_{v \in \mathbb{Z}^{2}}\left|a_{v}\right| \cdot\|b\|_{l^{1}}=\|a\|_{l^{1}} \cdot\|b\|_{l^{1}} .
$$

On the other hand, $\|a\| \leq \sum_{v \in \mathbb{Z}^{2}}\left\|a_{v} U_{v}\right\|=\|a\|_{l^{1}}$.

Convergence criteria. If one wants to solve the equation

$$
\delta_{\tau}(x)=x \delta_{\tau}(a)
$$

for given $a \in A_{\theta}$ one can try to consider the series

$$
x=\left.\boldsymbol{E}_{l}(\tau, a)\right|_{t=1}=1+a_{1}+a_{2}+\cdots
$$


obtained by substituting $t=1$ into $\boldsymbol{E}_{l}(\tau, a)=1+t a_{1}+t^{2} a_{2}+\cdots$. This series does not always converge even for $\theta=0$ (remember that in the commutative case it represents the function $\exp (t a) / \operatorname{tr}(\exp (t a)))$. One of our goals in this section is to show that (3-2) always converges for sufficiently small $a$.

Remark. In the commutative case the convergence improves when we switch from $\boldsymbol{E}_{l}(\tau, a)$ to $\operatorname{Exp}_{l}(\tau, a)$, since the latter series becomes the usual exponential. However, this does not seem to work in noncommutative case. Indeed, as we will see in Proposition 3.13(ii), there exists $a \in A_{\theta}$ for which (3-1) has no invertible solutions. By Proposition 2.6 this means that either $\left.\operatorname{Exp}_{l}(\tau, a)\right|_{t=1}$ or $\left.\operatorname{Exp}_{r}(\tau,-a)\right|_{t=1}$ diverges.

The next lemma follows from [Schweitzer 1993, Corollary 7.16], but since our particular case is much easier we present a more direct proof due to Chris Phillips.

Lemma 3.1. If $x \in A_{\theta}$ is invertible as an element of $\bar{A}_{\theta}$ then $x \in A_{\theta}^{*}$.

Proof. First, we claim that for every $a \in A_{\theta}$ such that $\|a\|<1$, the series $1+a+$ $a^{2}+\cdots$ converges to an element of $A_{\theta}$. Indeed, it suffices to show that it converges absolutely in every Sobolev space $W_{s}$. But this follows from the fact that for every $k$ there exists a constant $C$ (depending on $a$ ) such that $\left\|\delta^{k}\left(a^{n}\right)\right\| \leq C\|a\|^{n-k}$ for $n>k$. Thus, the assertion is true when $\|x-1\|<1$. The general case can be reduced to this by the following trick. Let $x$ be an element of $A_{\theta}$ such that there exists an inverse $x^{-1} \in \bar{A}_{\theta}$. Choose $y \in A_{\theta}$ such $\left\|y-x^{-1}\right\|<\|x\|^{-1}$. Then the element $x y \in A_{\theta}$ satisfies $\|x y-1\|<1$. Hence, $x y \in A_{\theta}^{*}$. It follows that $x$ has a right inverse in $A_{\theta}$. A similar argument shows that $y x \in A_{\theta}^{*}$ and hence $x$ has a left inverse. This implies that $x \in A_{\theta}^{*}$.

Remark. In fact, it is also true that if $x y=1$ in $A_{\theta}$ then $y x=1$. This follows from the existence of a finite positive faithful trace on $A_{\theta}$; see [Davidson 1996, second half of p. 101 and Exercises 6 and 9 of Chapter 4]. Here is another argument using Rieffel's classification of finitely generated projective $A_{\theta}$-modules in [Rieffel 1983]. We assume that $\theta$ is irrational. Let $I \subset A_{\theta}$ be the set of all $a$ such that $x a=0$. Then $I$ is a right ideal and we have $A_{\theta}=I \oplus y A_{\theta}$. It follows that $y A_{\theta}$ and $I$ are projective modules. Furthermore, $y A_{\theta}$ is isomorphic to $A_{\theta}$; hence rk $y A_{\theta}=1$. It follows that $\operatorname{rk} I=0$, so $I=0$.

Theorem 3.2. Let $d=\min \{|m \tau+n|: m, n \in \mathbb{Z},(m, n) \neq(0,0)\}$. For every $a \in A_{\theta}$ with $\operatorname{tr}(a)=0$ such that $\left\|\delta_{\tau}(a)\right\|<2 \pi d$, the series (3-2) converges to an element $\boldsymbol{e}_{l}(\tau, a) \in A_{\theta}$ satisfying the equation

$$
\delta_{\tau}\left(\boldsymbol{e}_{l}(\tau, a)\right)=\boldsymbol{e}_{l}(\tau, a) \delta_{\tau}(a) .
$$

Furthermore, if $\left\|\delta_{\tau}(a)\right\|_{l^{1}}<\pi d$ then $\boldsymbol{e}_{l}(\tau, a) \in A_{\theta}^{*}$. The map $a \mapsto \boldsymbol{e}_{l}(\tau, a)$ from $U_{\tau}=\left\{a \in H:\left\|\delta_{\tau}(a)\right\|_{l^{1}}<\pi d\right\}$ to $A_{\theta}^{*}$ is continuous. 
Proof. Recall that the $a_{i}$ are defined inductively by the conditions $\delta\left(a_{i}\right)=a_{i-1} \delta(a)$, $\operatorname{tr}\left(a_{i}\right)=0$, where $\delta=\delta_{\tau}$. It follows that

$$
\left\|a_{i}\right\|_{0} \leq(2 \pi d)^{-1}\left\|\delta\left(a_{i}\right)\right\|_{0} \leq(2 \pi d)^{-1}\|\delta(a)\| \cdot\left\|a_{i-1}\right\|_{0} .
$$

Hence, $\left\|a_{n}\right\|_{0} \leq(\|\delta(a)\| /(2 \pi d))^{n}$ for every $n \geq 0$. It follows that the series $1+$ $a_{1}+a_{2}+\cdots$ converges to an element $x$ in the $L^{2}$-completion of $A_{\theta}$. Furthermore, since

$$
\left\|\delta\left(a_{n}\right)\right\|_{0} \leq\|\delta(a)\| \cdot\left\|a_{n-1}\right\|_{0} \leq\|\delta(a)\| \cdot\left(\frac{\|\delta(a)\|}{2 \pi d}\right)^{n-1}
$$

we see that in fact $x$ belongs to the Sobolev space $W_{1}$ and satisfies the equation $\delta(x)=x \delta(a)$. This implies that $x \in A_{\theta}$.

To prove the last assertion we first observe that $\left\|a_{n}\right\|_{l^{1}} \leq r^{n}$ for all $n$, where $r=\|\delta(a)\|_{l^{1}} /(2 \pi d)$ (this is deduced in the same way as above). Hence,

$$
\left\|a_{1}+a_{2}+\cdots\right\| \leq\left\|a_{1}+a_{2}+\cdots\right\|_{l^{1}} \leq \frac{r}{1-r}<1 \quad \text { if } r<\frac{1}{2} .
$$

Therefore, $x$ is invertible in $\bar{A}_{\theta}$. It remains to apply Lemma 3.1.

The following result shows that our exponentials converge if all the coefficients of $a$ belong to closed half-planes not containing zero.

Proposition 3.3. Let $a=\sum_{v} a_{v} U_{v} \in A_{\theta}$ be an element with $\operatorname{tr}(a)=0$. Assume that there exists a homomorphism $h: \mathbb{Z}^{2} \rightarrow \mathbb{R}$ and a positive constant $\varepsilon$ such that $h>\varepsilon$ on $\operatorname{supp} a=\left\{v \in \mathbb{Z}^{2}: a_{v} \neq 0\right\}$. Then the series $1+a_{1}+a_{2}+\cdots$ converges to an element of $A_{\theta}^{*}$.

Proof. Since the elements $a_{i} \in H$ are defined inductively by $a_{1}=a$ and $\delta\left(a_{i}\right)=$ $a_{i-1} \delta(a)$, we have $h>n \varepsilon$ on $\operatorname{supp} a_{n}$. Let $d_{n}=\min _{(m, n): h(m, n)>n \varepsilon}|m \tau+n|$. Then

$$
\left\|a_{n}\right\|_{0} \leq\left(2 \pi d_{n}\right)^{-1}\left\|\delta\left(a_{n}\right)\right\|_{0} \leq\left(2 \pi d_{n}\right)^{-1}\|\delta(a)\| \cdot\left\|a_{n-1}\right\|_{0} .
$$

Since $d_{n}$ grows linearly with $n$ this immediately implies convergence of the series $\sum a_{n}$ in the $L^{2}$-norm. Arguing as in the proof of Theorem 3.2 we derive that $x=1+a_{1}+a_{2}+\cdots$ belongs to $A_{\theta}^{*}$.

Remark. In the situation of the proposition one has $\left.\operatorname{Exp}_{l}(\tau, a)\right|_{t=1}=\left.\boldsymbol{E}_{l}(\tau, a)\right|_{t=1}$. However, at present we do not know any criteria for convergence of $\left.\operatorname{Exp}_{l}(\tau, a)\right|_{t=1}$ similar to Theorem 3.2.

The discriminant hypersurface. Recall that $H \subset A_{\theta}$ is the set of elements $a$ such that $\operatorname{tr}(a)=0$. For every $a \in A_{\theta}$ consider the operator

$$
d_{a}:=\delta_{\tau}+\operatorname{ad}(a): A_{\theta} \rightarrow A_{\theta} .
$$

We are interested in the subset of $H$ given by

$$
\Theta=\Theta_{\tau}=\left\{a \in H: \operatorname{dim} \operatorname{ker} d_{a}>1\right\} .
$$


Since $\mathbb{C} \subset \operatorname{ker} d_{a}$, this is also the set of $a \in H$ such that $\operatorname{ker}\left(\left.d_{a}\right|_{H}\right) \neq 0$. We are going to show that $\Theta_{\tau}$ is a complex hypersurface in $H$ in the sense that it can be given locally as the zero set of a holomorphic function on $H$.

Lemma 3.4. For every $a \in A_{\theta}$ one has $\operatorname{ker} d_{a} \simeq \operatorname{Hom}_{\mathscr{C}}(E, E)$ and coker $d_{a} \simeq$ $\operatorname{Ext}_{\mathscr{b}}^{1}(E, E)$, where $E$ is the holomorphic bundle $\left(A_{\theta}, \bar{\nabla}_{a}:=\delta_{\tau}+a\right)$. Thus $d_{a}$ is a Fredholm operator of index zero. The same assertions are true if we replace $d_{a}$ by its extension $d_{a}: W_{s+1} \rightarrow W_{s}$ to a Sobolev space.

Proof. The identification of $\operatorname{ker} d_{0}$ with endomorphisms of $E$ in the category $\mathscr{C}$ follows from the definitions. The identification of $\operatorname{coker}\left(d_{0}\right)$ with $\operatorname{Ext}_{\mathscr{C}}^{1}(E, E)$ is constructed as in [Polishchuk and Schwarz 2003, Proposition 2.4]; by Serre duality, $\operatorname{Ext}_{\mathscr{C}}^{1}(E, E) \simeq \operatorname{Hom}_{\mathscr{C}}(E, E)^{*}$ [Polishchuk 2004]. The remaining assertions are proved using the same techniques as in [Polishchuk 2004, Theorem 2.8].

Let $\mathscr{F}_{0}(H)$ be the space of Fredholm operators of index zero on $W_{0}(H)$, where $W_{0}(H)=\left\{a \in W_{0}: \operatorname{tr}(a)=0\right\}$. Recall from [Quillen 1985; Furutani 2004] that there is a natural holomorphic line bundle $\mathscr{L}$ over $\mathscr{F}_{0}(H)$, called the determinant line bundle. By definition, $\mathscr{L}$ is trivial over each open set $\varkappa_{A} \subset \mathscr{F}_{0}(H)$, where $A: W_{0}(H) \rightarrow W_{0}(H)$ is a fixed trace class operator and $\bigcup_{A}$ consists of operators $T$ such that $T+A$ is invertible. The transition functions of $\mathscr{L}$ with respect to this open covering are of the form

$$
g_{A, B}(T)=\operatorname{det}_{F}\left(1+(A-B)(T+B)^{-1}\right)=\operatorname{det}_{F}\left((T+A)(T+B)^{-1}\right),
$$

where $A, B$ are trace class operators and $\operatorname{det}_{F}$ is the Fredholm determinant.

Let $\mathscr{L} \subset \mathscr{F}_{0}(H)$ be the complement of $\bigcup_{0}$, that is, the subset consisting of $T$ such that $\operatorname{ker} T \neq 0$. This is a complex hypersurface in $\mathscr{F}_{0}(H)$. More precisely, there exists a global holomorphic section $s$ of the determinant line bundle $\mathscr{L}$ such that $\mathscr{L}$ coincides with the zero locus of $s$. Under the standard trivialization of $\mathscr{L}$ over $U_{A}$ the section $s$ corresponds to the holomorphic function

$$
s_{A}(T)=\operatorname{det}_{F}\left(1-(T+A)^{-1} A\right) .
$$

One can easily check that $s_{B}=g_{A, B} s_{A}$, so these functions glue into a global section of $\mathscr{L}$. One has $s_{A}(T)=0$ if and only if $1-(T+A)^{-1} A$ has a nonzero kernel. But $1-(T+A)^{-1} A=(T+A)^{-1} T$, so $s_{A}(T)=0$ if and only if $\operatorname{ker} T \neq 0$. Thus the zero locus of $s$ coincides with $\mathscr{L}$.

We have a map

$$
H \rightarrow \mathscr{F}_{0}(H):\left.a \mapsto d_{a}\right|_{W_{0}(H)} .
$$

Note that the restriction of $d_{a}$ to $H$ (and to $W_{0}(H)$ ) still has index zero, since $d_{a}\left(A_{\theta}\right) \subset H$ and $\operatorname{ker} d_{a}=\mathbb{C} \oplus \operatorname{ker}\left(\left.d_{a}\right|_{H}\right)$. Abusing notation, we denote by $\mathscr{L}$ also the pull-back of the determinant line bundle to $H$ under the map above. 
Proposition 3.5. (i) The subset $\Theta \subset H$ coincides with the zero locus of the global holomorphic section $s\left(\left.d_{a}\right|_{W_{0}(H)}\right)$ of $\mathscr{L}$ over $H$.

(ii) For every one-dimensional subspace $L \subset H$ the intersection $\Theta \cap L$ is a discrete subset of $L \backslash\{0\}$.

Proof. (i) is clear since $\Theta$ is the preimage of $\mathscr{E} \subset \mathscr{F}_{0}(H)$. For (ii), observe that $\delta_{\tau}: W_{0}(H) \rightarrow W_{-1}(H)$ is an isomorphism and the operator $\operatorname{ad}(a): W_{0}(H) \rightarrow$ $W_{-1}(H)$ is compact for every $a \in A_{\theta}$; see the proof of [Polishchuk 2004, Theorem 2.8]. Hence, for every $a \in H$ and $t \in \mathbb{C}$ we have $t a \in \Theta$ if and only if $-t^{-1}$ belongs to the spectrum of the compact operator $\delta_{\tau}^{-1} \operatorname{ad}(a): W_{0}(H) \rightarrow W_{0}(H)$.

Remark. Proposition 3.5(ii) implies that $\Theta$ contains no linear subspaces of $H$. On the other hand, we will see later that $\Theta$ is swept by infinite-dimensional affine subspaces (see Theorem 3.17).

We can also consider more general loci

$$
\Theta_{\tau}^{(n)}=\left\{a \in H: \operatorname{dim} \operatorname{ker} d_{a}>n+1\right\}=\left\{a \in H: \operatorname{dim} \operatorname{ker}\left(\left.d_{a}\right|_{H}\right)>n\right\}
$$

for $n \geq 0$. For $n=0$ we get $\Theta_{\tau}^{(0)}=\Theta_{\tau}$. Since the kernel of $d_{a}$ coincides with the kernel of its extension to $W_{0}$, it follows that the loci $\Theta_{\tau}^{(n)}$ are closed.

Logarithmic derivative and connected components of groups of invertible elements. In this section we will study the relation between holomorphic structures and the group of invertible elements in $A_{\theta}$.

Theorem 3.6. Consider the map $L_{\tau}: A_{\theta}^{*} \rightarrow A_{\theta}: x \mapsto x^{-1} \delta_{\tau}(x)$.

(i) The composition

$$
\chi_{\tau}:=\operatorname{tr} \circ L_{\tau}: A_{\theta}^{*} \rightarrow \mathbb{C}
$$

is a locally constant homomorphism with image $(2 \pi i)(\mathbb{Z}+\mathbb{Z} \tau) \subset \mathbb{C}$ and kernel $\left(A_{\theta}^{*}\right)_{0}$, the connected component of 1 in $A_{\theta}^{*}$.

(ii) Let $H \subset A_{\theta}$ be the set of elements a such that $\operatorname{tr}(a)=0$. Let

$$
\Omega_{\tau}=\left\{a \in H: \operatorname{dim} \operatorname{ker}\left(\delta_{\tau}+\operatorname{ad}(a)\right)=1\right\}
$$

be the complement to the hypersurface $\Theta_{\tau} \subset H$. Then $\Omega_{\tau}$ is a dense open subset of $H$ and $L_{\tau}$ induces homeomorphisms

$$
A_{\theta}^{*} / \mathbb{C}^{*} \stackrel{\sim}{\longrightarrow} \Omega_{\tau}+2 \pi i(\mathbb{Z}+\mathbb{Z} \tau) \text { and }\left(A_{\theta}^{*}\right)_{0} / \mathbb{C}^{*} \stackrel{\sim}{\longrightarrow} \Omega_{\tau}
$$

Proof. From the Leibniz rule we immediately get

$$
L_{\tau}(x y)=y^{-1} L_{\tau}(x) y+L_{\tau}(y) .
$$

Taking traces we see that $\chi_{\tau}=\operatorname{tr} \circ L_{\tau}$ is a homomorphism. It is easy to see that the derivative of $L_{\tau}$ at the point $x \in A_{\theta}^{*}$ is $a \mapsto x^{-1} \delta_{\tau}\left(a x^{-1}\right) x$. Hence, the derivative 
of $\chi_{\tau}$ is $a \mapsto \operatorname{tr}\left(x^{-1} \delta_{\tau}\left(a x^{-1}\right) x\right)=0$. This implies that $\chi_{\tau}$ is locally constant. In particular, $\chi_{\tau}\left(\left(A_{\theta}^{*}\right)_{0}\right)=0$; that is, $L_{\tau}\left(\left(A_{\theta}\right)_{0}\right) \in H$.

Next we claim that the map $L_{\tau}:\left(A_{\theta}^{*}\right)_{0} / \mathbb{C}^{*} \rightarrow H$ is a local homeomorphism. Indeed, (3-3) shows that it is enough to check that $L_{\tau}$ is a local homeomorphism in a neighborhood of $1 \in\left(A_{\theta}^{*}\right)_{0} / \mathbb{C}^{*}$. Then we can use the map $\boldsymbol{e}_{l}$ constructed in Theorem 3.2 to get a local inverse to $L_{\tau}$.

We know that the space of holomorphic endomorphisms of $\left(A_{\theta}, \delta_{\tau}+a\right)$ can be identified with $\operatorname{ker}\left(\delta_{\tau}+\operatorname{ad}(a)\right.$ ) (see Lemma 3.4). Using the classification of holomorphic bundles on noncommutative tori given in [Polishchuk 2004] we derive that the dimension of this space is 1 if and only if there exists a holomorphic isomorphism of $\left(A_{\theta}, \bar{\nabla}\right)$ with a standard holomorphic bundle $\left(A_{\theta}, \delta_{\tau}+z\right)$ for some $z \in \mathbb{C}$. In other words, $\operatorname{ker}\left(\delta_{\tau}+\operatorname{ad}(a)\right)=\mathbb{C}$ if and only if there exists $x \in A_{\theta}^{*}$ such that $a \equiv L_{\tau}(x) \bmod \mathbb{C}$. Thus, the map

$$
A_{\theta}^{*} / \mathbb{C}^{*} \rightarrow H, \quad x \mapsto L_{\tau}(x)-\operatorname{tr}\left(L_{\tau}(x)\right)
$$

has $\Omega_{\tau}$ as its image. As we have seen, this map is a local homeomorphism, so $\Omega_{\tau}$ is open in $H$. Now we claim that the nonempty fibers of (3-4) are exactly the orbits of the action of the central subgroup $\mathbb{Z}^{2} \subset A_{\theta}^{*} / \mathbb{C}^{*}$. Indeed, assume that $L_{\tau}(x)=L_{\tau}(y) \bmod \mathbb{C}$ for some $x, y \in A_{\theta}^{*}$. Set $z=x y^{-1}$. Then

$$
L_{\tau}(x)=L_{\tau}(z y)=y^{-1} L_{\tau}(z) y+L_{\tau}(y),
$$

which implies that $y^{-1} L_{\tau}(z) y \in \mathbb{C}$; hence, $L_{\tau}(z) \in \mathbb{C}$. But this is possible only if $z$ is proportional to $U_{v}$ for some $v \in \mathbb{Z}^{2}$. This proves our claim about the fibers of the map (3-4).

Proposition 3.5(ii) immediately implies that $\Omega_{\tau}$ is connected (and dense in $H$ ). Since we have identified the quotient of $A_{\theta}^{*} / \mathbb{C}^{*}$ by $\mathbb{Z}^{2}$ with $\Omega_{\tau}$, it follows that the embedding $\mathbb{Z}^{2} \subset A_{\theta}^{*}$ induces an isomorphism on connected components. Since $\chi_{\tau}$ is locally constant, this implies that

$$
\chi_{\tau}\left(A_{\theta}^{*}\right)=\chi_{\tau}\left(\mathbb{Z}^{2}\right)=(2 \pi i)(\mathbb{Z}+\mathbb{Z} \tau) .
$$

We also see that $L_{\tau}:\left(A_{\theta}^{*}\right)_{0} / \mathbb{C}^{*} \rightarrow \Omega_{\tau}$ is a homeomorphism.

Corollary 3.7. For every $a \in H$ there exists a nonzero $x \in A_{\theta}$ such that $\delta_{\tau}(x)=a x$, and one such that $\delta_{\tau}(x)=x a$.

Proof. If $-a \in \Omega_{\tau}$, the preceding theorem says there exists $x \in A_{\theta}^{*}$ such that $\delta_{\tau}(x)=$ $-x a$. Therefore, $\delta_{\tau}\left(x^{-1}\right)=a x^{-1}$. If $-a \notin \Omega_{\tau}$, the holomorphic bundle $\left(A_{\theta}, \bar{\nabla}_{-a}\right)$ is decomposable (note that $\bar{\nabla}_{-a}=\delta_{\tau}-a$ ). Since one of the indecomposable factors must be of positive degree, this implies that $H^{0}\left(A_{\theta}, \bar{\nabla}_{-a}\right) \neq 0$. The case of the equation $\delta_{\tau}(x)=x a$ reduces to the previous case by using the identification $A_{\theta}^{\text {opp }}=A_{-\theta}$. 
As another corollary we get a new proof of the following well known fact.

Corollary 3.8. The embedding $\mathbb{Z}^{2} \subset A_{\theta}^{*}$ induces an isomorphism on connected components.

Using the results of [Polishchuk 2004] we can give one more characterization of the open subset $\Omega_{\tau} \subset H$. As in the discussion on pages 170-171 this leads to a set of local equations for its complement $\Theta_{\tau}$. For every $a \in H$, set $\bar{\nabla}_{a}=\delta_{\tau}+a$.

Proposition 3.9. For $a \in H$ one has $a \in \Omega_{\tau}$ if and only if there is $z \in \mathbb{C}$ such that

$$
\operatorname{ker}\left(\bar{\nabla}_{a+z}\right)=\left\{x: \delta_{\tau}(x)+(a+z) x=0\right\}
$$

is zero. Moreover, for $a \in \Omega_{\tau}$ this holds for all $z \notin 2 \pi i(\mathbb{Z}+\mathbb{Z} \tau)$. Hence, if we choose any $z_{0} \notin 2 \pi i(\mathbb{Z}+\mathbb{Z} \tau)$ then $a \in \Omega_{\tau}$ if and only if the operator $\bar{\nabla}_{a+z_{0}}$ is invertible.

Proof. If $a \in \Omega_{\tau}$ then this follows from the fact that cohomology of the standard holomorphic bundle $\left(A_{\theta}, \delta_{\tau}+z\right)$ vanishes for $z \notin 2 \pi i(\mathbb{Z}+\mathbb{Z} \tau)$. If $a \notin \Omega_{\tau}$, it suffices to observe that the holomorphic bundle $\left(A_{\theta}, \bar{\nabla}_{a+z}\right)$ has a direct factor of positive degree.

One can generalize some of the assertions of Theorem 3.6 to a slightly more general context.

Proposition 3.10. Let $(E, \bar{\nabla})$ be a basic right module over $A_{\theta}$ equipped with a holomorphic structure. Then the map

$$
\chi_{E}: \operatorname{Aut}_{A_{\theta}}(E) \rightarrow \mathbb{C}: x \mapsto \operatorname{tr}\left(x^{-1}[\bar{\nabla}, x]\right)
$$

is a locally constant homomorphism that does not depend on $\bar{\nabla}$. Its kernel coincides with the connected component of 1 in $\operatorname{Aut}_{A_{\theta}}(E)$ and its image is the lattice $(2 \pi i / \mathrm{rk} E)(\mathbb{Z}+\tau \mathbb{Z}) \subset \mathbb{C}$, where $\operatorname{rk} E \in(\mathbb{Z}+\mathbb{Z} \theta) \cap \mathbb{R}_{>0}$ is the rank of $E$. If $E=E^{\prime} \oplus E^{\prime \prime}$ is a decomposition into the direct sum of $A_{\theta}$-modules, where $E^{\prime}$ is also basic, one has the commutative diagram

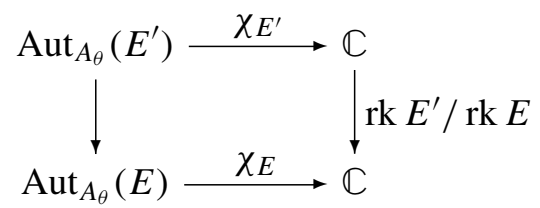

Proof. Any other holomorphic structure on $E$ has form $\bar{\nabla}+\phi$ for some $\phi$ in $\operatorname{End}_{A_{\theta}}(E)$. But

$$
\operatorname{tr}\left(x^{-1}[\bar{\nabla}+\phi, x]\right)=\operatorname{tr}\left(x^{-1}[\bar{\nabla}, x]\right)+\operatorname{tr}\left(x^{-1} \phi x-\phi\right)=\operatorname{tr}\left(x^{-1}[\bar{\nabla}, x]\right) .
$$

This shows that $\chi_{E}$ is independent of $\bar{\nabla}$. If we choose $\bar{\nabla}$ to be standard (see [Polishchuk 2004]), the pair $\left(\operatorname{End}_{A_{\theta}}\right.$, ad $\left.\bar{\nabla}\right)$ can be identified with a pair of the 
form $\left(A_{\theta^{\prime}}, \delta_{\tau} / r\right)$ for some $\theta^{\prime}$, where $r=\operatorname{rk} E$; see [Polishchuk and Schwarz 2003, Proposition 2.1]. It remains to apply Theorem 3.6.

Since the homomorphism $\chi_{E}$ does not depend on the choice of a holomorphic structure, we can choose a holomorphic structure on $E$ compatible with the decomposition $E=E^{\prime} \oplus E^{\prime \prime}$. Then commutativity of (3-5) follows from the compatibility of the embedding $i: \operatorname{End}_{A_{\theta}}\left(E^{\prime}\right) \rightarrow \operatorname{End}_{A_{\theta}}(E)$ with normalized traces:

$$
\operatorname{tr}(i(x))=\frac{\mathrm{rk} E^{\prime}}{\operatorname{rk} E} \operatorname{tr}(x) .
$$

As a corollary we get a new proof of the following well known fact.

Corollary 3.11. Let $E$ be a basic right module over $A_{\theta}$, and let $E=E^{\prime} \oplus E^{\prime \prime}$ be a decomposition into the direct sum of $A_{\theta}$-modules, where $E^{\prime}$ is also a basic module. Then the natural homomorphism $\operatorname{Aut}_{A_{\theta}}\left(E^{\prime}\right) \rightarrow \operatorname{Aut}_{A_{\theta}}(E)$ induces an isomorphism on connected components.

Proof. Apply commutative diagram (3-5) together with the fact that the image of $\chi_{E}$ is $2 \pi i \operatorname{rk}(E)(\mathbb{Z}+\mathbb{Z} \tau)$, and likewise for $E^{\prime}$.

Corollary 3.12. Let $E$ be a basic right module over $A_{\theta}$ and let $E=E^{\prime} \oplus E^{\prime \prime}$ be a decomposition into a direct sum of $A_{\theta}$-modules. Then the natural homomorphism Aut $_{A_{\theta}} E^{\prime} \rightarrow$ Aut $_{A_{\theta}}$ E induces a surjection on connected components.

Proof. It suffices to choose any decomposition $E^{\prime}=E_{1}^{\prime} \oplus E_{2}^{\prime}$ with $E_{1}^{\prime}$ basic and apply Corollary 3.11.

Remark. The embedding of $A_{\theta}$ into $\mathrm{Mat}_{n} A_{\theta}$ induces an isomorphism on connected components of groups of invertible elements. (For $\bar{A}_{\theta}$ this statement is [Rieffel 1983, Theorem 8.3]; the case of $A_{\theta}$ follows using its invariance under the holomorphic functional calculus.) This allows to deduce from Corollary 3.11 the same statement for $E^{\prime}$ not necessarily basic.

More on the discriminant hypersurface. It would be interesting to study intersections of $\Theta_{\tau}$ with finite-dimensional subspaces of $H$. In the following proposition we consider simplest examples.

Proposition 3.13. (i) Let $h: \mathbb{Z}^{2} \rightarrow \mathbb{R}$ be a homomorphism. Assume that $a \in H$ is an element such that $h>\varepsilon$ on supp a for some $\varepsilon>0$. Then $\mathbb{C} a \in \Omega_{\tau}$. The same conclusion holds if we assume that $h$ takes values in $\mathbb{Q}$ and that $h \geq 0$ on $\operatorname{supp} a$.

(ii) Let $e \in A_{\theta}$ be a nontrivial idempotent such that $\delta_{\tau}$ preserves $e A_{\theta} \subset A_{\theta}$, i.e., $e \delta_{\tau}(e)=\delta_{\tau}(e)$. Then $\Theta_{\tau} \cap \mathbb{C} \delta_{\tau}(e)=\left\{\delta_{\tau}(e)\right\}$.

Lemma 3.14. Let $a=x_{0}^{-1} \delta_{\tau}\left(x_{0}\right)$ for some $x_{0} \in A_{\theta}^{*}$. Then every $x \in A_{\theta}$ such that $\delta_{\tau}(x)=x a$ is proportional to $x_{0}$. 
Proof. Indeed, $\delta\left(x x_{0}^{-1}\right)=\delta(x) x_{0}^{-1}-x x_{0}^{-1} \delta\left(x_{0}\right)^{-1} x_{0}^{-1}=0$. Hence $x x_{0}^{-1}$ is a constant.

Proof of Proposition 3.13. (i) It suffices to show that the equation $L_{\tau}(x)=a$ has a solution with $x \in A_{\theta}^{*}$. If $h>\varepsilon$ on supp $a$, this follows from Proposition 3.3. If $h$ takes values in $\mathbb{Q}$ and $h \geq 0$ on $\operatorname{supp} a$, we can write $a=b+c$, where $h=0$ on $\operatorname{supp} b$ and $h>\varepsilon>0$ on $\operatorname{supp} c$. Since the subalgebra of elements supported on $h=0$ is commutative and stable under $\delta_{\tau}$, we have $b=L_{\tau}(x)$ for some $x \in A_{\theta}^{*}$ such that $h=0$ on $\operatorname{supp} x$. Now consider the element $c^{\prime}=x c x^{-1}$. Note that $h>0$ on $\operatorname{supp} c^{\prime}$. Therefore, by Proposition 3.3, there exists an element $y \in A_{\theta}^{*}$ such that $c^{\prime}=L_{\tau}(y)$. Then

$$
L_{\tau}(y x)=x^{-1} L_{\tau}(y) x+L_{\tau}(x)=x^{-1} c^{\prime} x+b=c+b=a .
$$

(ii) For $\lambda \in \mathbb{C}^{*}$ consider the element $x_{\lambda}=\lambda e+(1-e) \in A_{\theta}$. Then $x_{\lambda} \in\left(A_{\theta}^{*}\right)_{0}$ and $x_{\lambda}^{-1}=\lambda^{-1} e+(1-e)$. Hence

$$
L_{\tau}\left(x_{\lambda}\right)=\left(\lambda^{-1} e+(1-e)\right)(\lambda-1) \delta_{\tau}(e)=\left(1-\lambda^{-1}\right) \delta_{\tau}(e) .
$$

It follows that $z \delta_{\tau}(e) \in \Omega_{\tau}$ for all $z \in \mathbb{C} \backslash\{1\}$. Now let us prove that $\delta_{\tau}(e) \notin \Omega_{\tau}$. Assume that there exists $x_{0} \in A_{\theta}^{*}$ such that

$$
x_{0}^{-1} \delta_{\tau}\left(x_{0}\right)=\delta_{\tau}(e) .
$$

Since we also have $\delta_{\tau}(e)=e \delta_{\tau}(e)$, applying Lemma 3.14 to $x=e$ we derive that $e$ should be proportional to $x_{0}$. Since $e$ is not invertible we get a contradiction.

Remark. There are plenty of idempotents $e \in A_{\theta}$ such that $e A_{\theta}$ is preserved by $\delta_{\tau}$. In fact, for every number $0<m \theta+n<1$ such that $m, n \in \mathbb{Z}$ and $m<0$ there exists an idempotent $e$ as above with $\operatorname{tr}(e)=m \theta+n$; see [Polishchuk $\geq 2006$ ].

Next we describe the decomposition of the discriminant locus $\Theta_{\tau} \subset H$ corresponding to types of idempotents in $A_{\theta}$.

Lemma 3.15. Assume that $\theta$ is irrational. Two idempotents $e$ and $e^{\prime}$ in $A_{\theta}$ are conjugate by an element in $\left(A_{\theta}^{*}\right)_{0}$ if and only if $\operatorname{tr}(e)=\operatorname{tr}\left(e^{\prime}\right)$.

Proof. The "only if" part is trivial. Assume that $\operatorname{tr}(e)=\operatorname{tr}\left(e^{\prime}\right)$. By Rieffel's classification of projective $A_{\theta}$-modules (see [Rieffel 1983]), there exist isomorphisms of right $A$-modules $e A \simeq e^{\prime} A$ and $(1-e) A \simeq\left(1-e^{\prime}\right) A$. Therefore, there exists an element $x \in A_{\theta}^{*}$ such that $x e A=e^{\prime} A$ and $x(1-e) A=\left(1-e^{\prime}\right) A$. This immediately implies the equality of the idempotents $x e x^{-1}=e^{\prime}$. It remains to show that $x$ can be chosen in $\left(A_{\theta}^{*}\right)_{0}$. But this follows easily from Corollary 3.12.

For every idempotent $e$, denote by $A_{e, \tau}$ the set of $a \in A_{\theta}$ such that the operator $x \mapsto \delta(x)+a x$ preserves the decomposition $A_{\theta}=e A_{\theta} \oplus(1-e) A_{\theta}$ :

$$
A_{e, \tau}=\left\{a \in A_{\theta}: \delta(e)+a e \in e A_{\theta}, \delta(1-e)+a(1-e) \in(1-e) A_{\theta}\right\} .
$$


Equivalently, $a \in A_{e, \tau}$ if and only if $a$ satisfies the equations

$$
e \delta(e)=e a(1-e) \quad \text { and } \quad(1-e) a e=(1-e) \delta(1-e) .
$$

This shows that $A_{e, \tau}$ is an affine subspace in $A_{\theta}$ with the associated linear subspace $e A e+(1-e) A(1-e)$.

Lemma 3.16. For $x \in A_{\theta}^{*}$ and for an idempotent $e \in A_{\theta}$ the map $a \mapsto x^{-1} a x+L_{\tau}(x)$ induces a bijection from $A_{e, \tau}$ to $A_{x^{-1} e x, \tau}$.

Proof. The natural action of $A_{\theta}^{*}$ on operators of the form $\delta_{\tau}+a$ is equivalent to the following action of $A_{\theta}^{*}$ on $A_{\theta}$ :

$$
x * a=x a x^{-1}+L_{\tau}\left(x^{-1}\right),
$$

where $x \in A_{\theta}^{*}, a \in A_{\theta}$. It is clear from the definition that the action of $x^{-1}$ sends $A_{e, \tau}$ to $A_{x^{-1} e x, \tau}$.

We refer to the action of $A_{\theta}^{*}$ on $A_{\theta}$ introduced above as the twisted action. Note that it preserves $H$ and $\Theta_{\tau}$.

Set $H_{e, \tau}=A_{e, \tau} \cap H$. For any $r \in(0,1) \cap(\mathbb{Z}+\mathbb{Z} \theta)$, set $\mathscr{H}_{r, \tau}=\bigcup_{e: \operatorname{tr} e=r} H_{e, \tau}$, where the union is taken over the set of all idempotents $e$ with $\operatorname{tr}(e)=r$. Since $A_{1-e, \tau}=A_{e, \tau}$ we have $\mathscr{H}_{1-r, \tau}=\mathscr{H}_{r, \tau}$.

Theorem 3.17. Assume that $\theta$ is irrational. Then

$$
\Theta_{\tau}=\bigcup_{r \in(0,1 / 2) \cap(\mathbb{Z}+\mathbb{Z} \theta)} \mathscr{H}_{r, \tau} .
$$

This decomposition is irreducible, in the sense that none of the subsets is contained in the union of the rest. If $e \in A_{\theta}$ is any idempotent with $\operatorname{tr}(e)=r$, we have a map

$$
\psi_{e}:\left(A_{\theta}^{*}\right)_{0} \times H_{e, \tau} \rightarrow H, \quad(x, a) \mapsto x^{-1} a x+L(x)
$$

such that $\mathscr{H}_{r, \tau}$ is the image of $\psi_{e}$. The codimension of the differential of $\psi_{e}$ at a point $(x, a)$ is $\operatorname{dim}_{\operatorname{Hom}_{\mathscr{C}}}\left(E_{1}, E_{2}\right)+\operatorname{dim}_{\operatorname{Hom}_{\mathscr{C}}}\left(E_{2}, E_{1}\right)$, where $\mathscr{C}$ is the category of holomorphic bundles on $T_{\theta, \tau}$ and $E_{1}, E_{2}$ are the summands of the holomorphic decomposition $\left(A_{\theta}, \delta_{\tau}+\psi(x, a)\right)=E_{1} \oplus E_{2}$ corresponding to the idempotent $x^{-1} e x$.

Proof. From the main theorem in [Polishchuk 2004] we know that a holomorphic bundle $E$ on a noncommutative torus has nonscalar endomorphisms then it is either decomposable or its rank is a nonprimitive element of $\mathbb{Z}+\mathbb{Z} \theta$. Since for a holomorphic bundle $E_{a}=\left(A_{\theta}, \delta_{\tau}+a\right)$ the second alternative is impossible, we conclude that $a \in \Theta_{\tau}$ if and only if $E_{a}$ is decomposable. This implies (3-6). Since $\psi_{e}$ is $\left(A_{\theta}^{*}\right)_{0}$-equivariant with respect to the twisted action on $H$ and the action by 
left multiplication on $\left(A_{\theta}^{*}\right)_{0}$, it suffices to consider the differential of $\psi_{e}$ at a point $\left(1, a_{0}\right)$, where $a_{0} \in H_{e}$. This differential maps

$(x, a) \in A_{\theta} \oplus H \cap(e A e+(1-e) A(1-e)) \quad$ to $\quad \delta_{\tau}(x)+\left[a_{0}, x\right]+a=d_{a_{0}}(x)+a$.

But by Lemma 3.4, $\operatorname{coker}\left(d_{a_{0}}: A_{\theta} \rightarrow A_{\theta}\right)$ is isomorphic to $\operatorname{Ext}_{\mathscr{C}}^{1}(E, E)$, where $E=\left(A_{\theta}, \delta_{\tau}+a_{0}\right)$. Furthermore, it is easy to see that the map $d_{a_{0}}$ is compatible with the decomposition

$$
A_{\theta}=e A_{\theta} e+(1-e) A_{\theta}(1-e) \oplus e A_{\theta}(1-e) \oplus(1-e) A_{\theta} e
$$

and that the cokernel of $d_{a_{0}}$ acting on each of these four pieces is identified with $\operatorname{Ext}^{1}\left(E_{i}, E_{j}\right)$, where $E=E_{1} \oplus E_{2}$ is the holomorphic decomposition induced by $e$. The assertion follows immediately.

Let $r_{0}$ be the unique number of the form $\pm \theta+n$ with $n \in \mathbb{Z}$ such that $0<r_{0}<1 / 2$. Proposition 3.18. (i) $\Theta_{\tau}^{(1)}=\Theta_{\tau}$.

(ii) $\Theta_{\tau}^{(2)}=\bigcup_{r \in(0,1 / 2) \cap(\mathbb{Z}+\mathbb{Z} \theta), r \neq r_{0}} \mathscr{H}_{r, \tau}$.

(iii) The natural map $\bigsqcup_{e: \operatorname{tr}(e)=r_{0}} H_{e, \tau} \backslash \Theta_{\tau}^{(2)} \rightarrow \Theta_{\tau} \backslash \Theta_{\tau}^{(2)}$ is an affine line bundle.

Proof. (i) follows from the fact that if $E_{a}$ is decomposable then $\operatorname{dim}_{\operatorname{End}}\left(E_{a}\right)>2$. Moreover, the only case when $\operatorname{dim}_{E_{c}}\left(E_{a}\right)=3$ is the following: $E_{a} \simeq E_{1} \oplus E_{2}$ in the holomorphic category, where $E_{1}$ and $E_{2}$ are simple, $\operatorname{Hom}_{\mathscr{C}}\left(E_{2}, E_{1}\right)=0$ and $\operatorname{Hom}_{\mathscr{C}}\left(E_{1}, E_{2}\right)$ is one-dimensional. Let $\mathrm{rk} E_{1}=m \theta+n$. Then $\mathrm{rk} E_{2}=-m \theta+$ $(1-n)$ and hence, by [Polishchuk 2004, Corollary 2.9],

$$
\operatorname{dim} \operatorname{Hom}_{\mathscr{C}}\left(E_{1}, E_{2}\right)-\operatorname{dim}_{\operatorname{Hom}_{\mathscr{C}}}\left(E_{2}, E_{1}\right)=-m n-m(1-n)=-m .
$$

This implies that $m=-1$, so either rk $E_{1}=r_{0}$ or rk $E_{2}=r_{0}$, which proves (ii). It remains to note that the set of all possible holomorphic decomposition of $E_{a}$ into $E_{1}$ and $E_{2}$ is a principal homogeneous space for $\operatorname{Hom}_{\mathscr{C}}\left(E_{1}, E_{2}\right)$, which leads to (iii).

The part of Theorem 3.17 concerning tangent maps should imply that $\mathscr{H}_{r, \tau}$ has (in an appropriate sense) codimension $\geq 2$ for $r \neq r_{0}$ (where $0<r<1 / 2$ ) while $\mathscr{H}_{r_{0}, \tau}$ is irreducible of codimension 1 . If we were in the finite-dimensional situation we would immediately deduce from this that the hypersurface $\Theta_{\tau}$ is irreducible. Our lack of knowledge does not allow us to state this precisely. However, we conjecture that the statements above hold when we intersect these loci with generic finite-dimensional subspaces in $A_{\theta}$.

\section{Acknowledgment}

I am grateful to Chris Phillips for many useful conversations and to Hanfeng Li for correcting a mistake in the first draft. 


\section{References}

[Davidson 1996] K. R. Davidson, $C^{*}$-algebras by example, Fields Institute Monographs 6, American Mathematical Society, Providence, RI, 1996. MR 97i:46095 Zbl 0958.46029

[Furutani 2004] K. Furutani, “On the Quillen determinant”, J. Geom. Phys. 49:3-4 (2004), 366-375. MR 2005i:58040 Zbl 1078.53077

[Polishchuk 2004] A. Polishchuk, "Classification of holomorphic vector bundles on noncommutative two-tori”, Doc. Math. 9 (2004), 163-181. MR 2005c:58013 Zbl 1048.32012

[Polishchuk $\geq 2006$ ] A. Polishchuk, "Quasicoherent sheaves on complex noncommutative two-tori", in preparation.

[Polishchuk and Schwarz 2003] A. Polishchuk and A. Schwarz, "Categories of holomorphic vector bundles on noncommutative two-tori”, Comm. Math. Phys. 236 (2003), 135-159. MR 2004k:58011 Zbl 1033.58009

[Quillen 1985] D. Quillen, "Determinants of Cauchy-Riemann operators on Riemann surfaces", Funktsional. Anal. i Prilozhen. 19:1 (1985), 37-41. In Russian; translated in Funct. Anal. Appl. 19 (1985), 31-34. MR 86g:32035 Zbl 0603.32016

[Rieffel 1983] M. A. Rieffel, "The cancellation theorem for projective modules over irrational rotation $C^{*}$-algebras", Proc. London Math. Soc. (3) 47:2 (1983), 285-302. MR 85g:46085 Zbl 0541. 46055

[Schweitzer 1993] L. B. Schweitzer, "Spectral invariance of dense subalgebras of operator algebras", Internat. J. Math. 4:2 (1993), 289-317. MR 94i:46097 Zbl 0804.46081

Received September 2, 2004.

Alexander PolishchuK

DEPARTMENT OF MATHEMATICS

UNIVERSITY OF OREGON

EUGENE, OR 97403

UNITED STATES

apolish@uoregon.edu 